\title{
Relativistic Modeling for the Giant Atom as a Key for Physics of Self Replication
}

\author{
Emad Eldieb
}

Forensic Medicine Organization, Egypt

Copyright $\bigcirc 2016$ by authors, all rights reserved. Authors agree that this article remains permanently open access under the terms of the Creative Commons Attribution License 4.0 International License

\begin{abstract}
In this paper we put the relativistic basis for the giant atom like system. Before we go with the discussion we have to stress on that it may appear in the beginning of this long study that there was a contradiction with stable facts like the constant speed of light and like quantum physics but as soon as possible we would find that the study completely respects such facts. Also we have to remember all over this study that the final macroscopic state of the micro scoping events inside the giant charge is the zero energy except from a little cut.
\end{abstract}

Keywords Giant Atom, Zero Energy, Quantum Physics, Self-replication

\section{Introduction}

From the relativistic relation $m^{2}=m_{o}^{2} / 1-\beta^{2}$ where $\beta=V / c$, if the speed $V$ of a particle $=c$ then the relativistic mass $\mathrm{m}$ would be infinite and if $V^{2}=2 c^{2}$ then, $m^{2}=-m_{o}^{2}$. Both are refused [1].

The Einstein famous equation [2] of the rest mass of a subatomic particle like a proton (or neutron....) is defined by;

$$
\left(k \frac{e^{2}}{r_{o}}\right)=m_{o} c^{2} \text {. Where it is a positive energy expresses }
$$

about the inertial $E_{o}$ such that $m=m_{o}$. If we multiply the left side (where $k$ denotes the electrostatic constant $=8.98 \times 10^{9} \mathrm{~N} \cdot \mathrm{m}^{2} / \mathrm{c}^{2}$ and $e$ is the value of the elementary charge in coulomb) by one then the right side should be typically the same (kindly look at the appendix number 1) as; $\left(k \frac{e^{2}}{r_{o}}\right)[1]=m_{o} c^{2}$. Where it is a positive inertial $E_{o}$ not else and $m=m_{o}$ (no motion). On the same form if we defined a system formed of a quantity of protons $p$, distributed homogeneously among much more number of neutrons $\mathrm{n}$, such that $n / p$ is defined (neglect quantum mechanics for a moment) and if we defined the quantity;

$$
\begin{aligned}
& {\left[\frac{r_{o}}{r_{c}} \cdot \frac{p^{2 / 3}}{\left(\frac{n}{p}\right)^{1 / 3}}\right]=[1] } \\
\therefore & \left(k \frac{e^{2}}{r_{o}}\right)\left[\frac{r_{o}}{r_{c}} \cdot \frac{p^{2 / 3}}{\left(\frac{n}{p}\right)^{1 / 3}}\right]=m_{o} c^{2}
\end{aligned}
$$

Where $m=m_{o}+0=m_{o}$

(If -and only if- the products inside the big brackets $=1$ ). But actually we can write eq. 1 in the form of the kinetic energy $\Delta m c^{2}=k e^{2} p / r$ which when put with eq.1 we get; $\Delta m c^{2}=m_{o} c^{2}$ which means that each defined proton of the sphere have gained $\Delta E=m_{o} c^{2}$ or literally each a proton have produced another one which is equivalent to; $\Delta m c^{2}+$ $m_{o} c^{2}=m c^{2}=2 m_{o} c^{2}$ or equivalently each particle is in the form of $m c^{2}=m_{o} 2 c^{2}$ which finally means that the inertial particle gained speed $=c$ which is -in relativityrefused and so eq. 1 becomes nonsense except it carries information that it can produce particles by a vague process. Now to overcome the problem in this refused equation and to initiate the action of physics of the sphere let the speed $V$ of its particles be close to $c$ so let us define $V$ when extremely approaches $c$ (but not equal $c$ ) as;

$$
V^{2}=c^{2}-c^{2} \frac{n}{p \times 1.29 \times 10^{36}} \text { where } n / p \text { is a }
$$

constant equal the ratio of the number of the neutrons to that of the proton in our sphere.

Let; $\beta^{2}=V^{2} / c^{2}$ and let;

$$
\begin{gathered}
1-\beta^{2}=1-1+\left(\frac{n}{p \times 1.29 \times 10^{36}}\right)=\frac{n}{p \times 1.29 \times 10^{36}}= \\
\frac{n}{p \cdot \mu}
\end{gathered}
$$




$$
\therefore m^{2}=c_{o}\left(m_{o}\right)^{2}=\mu \frac{p}{n} m_{o}^{2}=\left(1.29 \times 10^{36} \times \frac{p}{n}\right) m_{o}^{2}
$$

In the same sphere if each nucleon had such high speed (due to the relative positions $=r_{c}$ ) then the gravitational potential potential as speed function $u_{g}(v)$-from side of view of relativity for an imagined person localized at the centre of the sphere where he realizes the microscopic motive events - would be affected by the quantum speed $V$ more than the electric potential of each a proton $u_{e}(v)$ such that $1 / c_{o}^{1 / 2}$

$=\left[1-\left(V^{2} / c^{2}\right)\right]^{1 / 2}$ would appear in the denominator of $u_{g}(v)$ with power $3 / 2$ while it appears in the denominator of $u_{e}(v)$ only with power $1 / 2$.

$u_{g}(v)=-G n\left(m_{o}^{2} c_{o}\right)\left(c_{o}^{1 / 2} / r_{s}\right)$. While,

$u_{e}(v)=k e^{2} p\left(c_{o}^{1 / 2} / r_{s}\right)$. Where; $r_{s}$ is the radius of the sphere in a rest form.

$$
\left(k \frac{e^{2}}{r_{o}}\right)\left[\frac{r_{o}}{r_{c}} \cdot \frac{p^{2 / 3}}{\left(\frac{n}{p}\right)^{1 / 3}}\right]=\left(-G C_{o} \frac{m_{o}^{2}}{r_{o}}\right)\left(\frac{r_{o}}{r_{c}}\right) n^{2 / 3}
$$

Where; $c_{o}$ is the equality factor (defined from equation 2) and the negative sign means that the two sides act opposite to each other. The solution is in the form of asymmetric addition as;

$$
A+-A=0
$$

Let us search in other solution and so go back again;

$$
E_{o}=\left(k \frac{e^{2}}{r_{o}}\right)=m_{o} c^{2} \text { is an inertial positive rest }
$$

energy. And

$$
\left(k \frac{e^{2}}{r_{o}}\right)=\left(k \frac{e^{2}}{r_{o}}\right)\left[\frac{r_{o}}{r_{c}} \cdot \frac{p}{\left(\frac{n}{p} \cdot p\right)^{1 / 3}}\right] \text { is refused. }
$$

Let us recall the equality $\left(k e^{2} / r_{o}\right)[1]=-G c_{o} m_{o}^{2} n / r_{s}$

Put $\left(-m^{2}\right)$ as a relativistic equivalent to $m_{o}^{2}$ and substitute in the last form of the equality as;

$$
\begin{gathered}
\left(-m^{2}\right)=m_{o}^{2} \\
-G c_{o}\left(-m^{2} n\right) / r_{s}=\left(k \frac{e^{2}}{r_{o}}\right)\left[\frac{r_{o}}{r_{c}} \cdot \frac{p}{(n)^{1 / 3}}\right] \\
-G c_{o}\left(m^{2} n\right) / r_{s}=-\left(k \frac{e^{2}}{r_{o}}\right) \\
G c_{o}\left(m^{2} n\right) / r_{s}=\left(k \frac{e^{2}}{r_{o}}\right)[1]=\left(k \frac{e^{2}}{r_{o}}\right)=m_{o} c^{2} .
\end{gathered}
$$

The middle side -like the left- has become positive energy; such that each side is -now- having the same magnitude and the same positive form $0 \mathrm{f} ;\left(k \frac{e^{2}}{r_{o}}\right)$. The equality has -nowsymmetric additive solution as;

$$
u=m_{o} c^{2}+m_{o} c^{2}=2 m_{o} c^{2}=m_{o} 2 c^{2}
$$

This final solution says that each of the three sides of eq. 7 is not actually potential (not to bind the particles together) but explicitly an inertial energy where the sphere is particles factory.

The equality $\left(-m^{2}=m_{o}^{2}\right)$ has a relativistic solution in the form of;

$$
m^{2}=m_{o}^{2} / 1-\beta^{2}=m_{o}^{2} / 1-2=-m_{o}^{2}
$$

Which gives a relativistic solution as; $V^{2}=2 c^{2}$

This is the condition required for existence of the giant charge (or generally for the productive function) where such state does not mean $V>c$ but definitely means particles production. And the defined continuous production of the particles (as we will see) guarantees firstly that $m^{2}=m_{o}^{2}$ such that equation 2 would have the meaning of multiplication of the particles number by the factor $c_{o}$ instead of increase the mass of the particle by the same factor where we are in a rest state (except from little residual energy as we will see) and secondly that the speed $\mathrm{c}$ is a universal constant. This means that the additive relation of $V^{2}=c^{2}+$ $c^{2}$ (of eq.10) should be understood through the concept of eq. 8 (concept of production of two particles added to each particle) and the negative of $\mathrm{m}^{2}$ does not mean direction but it is an operator has a radical physical meaning such that it converted the kinetic electric and gravitational energies in the above equality into inertial energy. The same equalityafter understanding its negative sign- leaves behind the meaning;

$$
m^{2}=m_{o}^{2} \text { (and consequently } r=r_{o} \text { ) }
$$

(That is if only -and only if- the two potentials of eq. 1 are equal). And the equality of eq.11 explains the equality of eq. 10 where each means that the sphere is in rest motion and the speed which appears is the inertial $\mathrm{c}$ of the produced particles. Now if we recall the refused equality in eq. 1 and conclude it from eq. 8 and 9 then it would become;

$$
\left(k \frac{e^{2}}{r_{o}}\right)\left[\frac{r_{o}}{r_{c}} \cdot \frac{p}{\left(\frac{n}{p} \cdot p\right)^{1 / 3}}\right]=2 m_{o} c^{2} / 2=m_{o} c^{2}
$$

(The left side of this equality when put as a pair like the relations in equations 7 and 8 gives with its conjugate the output;

$$
\left.u \approx m_{o} c^{2}+m_{o} c^{2} \approx 2 m_{o} c^{2}\right)
$$

These approximate relations because of the speed of a particle never equal $\mathrm{c}$ but may be $\mathrm{v} \approx \mathrm{c}$. So let us again expect 
speed $\mathrm{v}$ in the form;

$$
V^{2}=c^{2}-c^{2} / c_{o}=c^{2}-c^{2} \frac{n}{p \times 1.29 \times 10^{36}}
$$

And let us put the relativistic mass as; $m^{2}=m_{o}^{2} / 1-\beta^{2}$ such that $\beta^{2}=V^{2} / c^{2}$ and $1-\beta^{2}=1 / c_{o}=1-1+$ $\left(\frac{n}{p} 1.29 \times 10^{36}\right)=\left(\frac{n}{p} 1.29 \times 10^{36}\right)$

Giving; $m^{2}=\left(1.29 \times 10^{36} \frac{p}{n}\right) m_{o}^{2}$. So we have to modify the relation of $V^{2}=2 c^{2}$ of the pair conjugate of eq.7 into $V^{2} \approx 2 c^{2}$ or

$$
V^{2}=2\left[c^{2}-\left(c^{2} \frac{n}{p \times 1.29 \times 10^{36}}\right)\right] \text { which instead of }
$$

the equality of eq. 11 would give the approximate relation; $m^{2} \approx m_{o}^{2}$. The factor $c_{o}$ which appears in the equality of eq.7 appears as the alternative relativistic solution (instead of $m^{2}=1.29 \times 10^{36} \frac{p}{n} m_{o}^{2}$ ) under the pressure of the following realized facts; first $m \approx m_{o}$ and second the necessary equality of the two potentials (or precisely; the two inertial) of eq.7. This means that; the gravitational constant appears as; in the form of eq. 6 if and only if $u_{G}$ has the meaning of inertia or explicitly; the triple $u_{G}, u_{e}$ and $u_{c}\left(u_{c}\right.$ is the quantum term as we will see soon) all are one thing called $m_{o} c^{2}$.

The quantity $m^{2}$ of the function $m_{(V)}^{2}=$ $m_{o}^{2} / 1-\beta^{2} \quad$ conserved

$m_{(V)}^{2}=\mu m_{o}^{2}=C_{o(V)} m_{o}^{2} n / p=m_{o}^{2} N / p$. where;

$V^{2}=2\left[c^{2}-\left(c^{2} \frac{n}{p \times 1.29 \times 10^{36}}\right)\right] \approx 2 c^{2}$

Now the relativistic solution of; $\left(k e^{2}\right)=G \mu m^{2}$ (at $V^{2}$ extremely approaches $c^{2}$ ) is conserved by the following relativistic alternative solution.

$\left(k e^{2}\right) p=G c_{o} m_{o}^{2} n=G m^{2}\left(n . c_{o}\right)$. Where; $m=m_{o}$.

And the physical meaning of $n . c_{o}$ appears from;

$\left(k e^{2} p\right)=G m^{2} N$. Where;

$\mu=c_{o}\left(\frac{n}{p}\right)=\left(c_{o} n\right) / p=\frac{N}{p}$. Or explicitly;

$$
\mu=c_{o}\left(n_{(t, x i)} / p_{(t, x i)}\right)=N / p_{(t, x i)}
$$

The meaning of $N$ appears - as a macroscopic picture for an imagined person who is looking for a rest reference frame where the global view of the microscopic motive events appear as conjugate rest energy - from the next idea;

Actually the sphere gives the solution as growth -with time $t$ - in the dimensional place $x i$ from the initial sphere with the initial number of particles $n$ and $p$ at $(0, x)$ let us call it the giant charge unit (GCU) till it reaches to the final sphere (let us call it the giant charge GC) with the final number of particles $N$ and $P$. Or;

$(G C U) c_{o}=(G C)$. We would expect also that when GCU grows till reaches GC, the suitable solution would be in the form of the asymmetric addition of eq.4 (kindly look at our paper; the electrostatic energy in a rest form).

Then;

$$
m^{2}=\mu\left(m_{o}\right)^{2}=1.29 \times 10^{36} m_{o}^{2}=\left(\frac{n}{p} c_{o}\right) m_{o}^{2}=\left(\frac{N}{p}\right) m_{o}^{2} .
$$

In this equation we notice that each of $\mathrm{m}$ and $m_{o}$ appears in a distinct specific position although they are equal and can alternate their positions. But this form where $\mathrm{m}$ appears in the first left side carries with the second left side the relativistic relation with $m_{o}$. While it carries with the third left side the alternative solution in GCU. And finally carries with the right side the alternative solution in the final sphere (GC). This alternative solution keeps $m=m_{o}$ which gives information that the sphere is in a rest state. The energy looks as if it turned from the region of the kinetic energy and flashed in the region of the inertial rest energy with the simple conservative solution;

a- conservative momentum; $P=P_{1}+P_{2}=0$

b- Conservative energy; $\int p d v=$ constant $=m 2 c^{2}$.

The addition may be in the form of;

$u \approx m\left(c^{2}+c^{2}\right) \approx m 2 c^{2}$ which realizes the state of $V^{2} \approx 2 c^{2}$. Or;

$u \approx c^{2}(m+m) \approx 2 m c^{2}$ which realizes the symmetric additive solution of the particles production.

Now the Gcu is formed of two distinguishable particles: the protons $p$ and the neutrons $n$ where $n \gg p$. So, each of both has its specific distinguishable potential (inertial) well[3]. We saw that the proton in its potential well appeared as a pair of inertial conjugate: $u_{e}+u_{G}$ [where $u_{G}=u_{g} c_{o}$ appears if (and only if) $u_{G}=u_{e}$ ].

Also the neutron does.

Let us define the Compton reduced wave length[4]; $r_{c} \approx 2 \times 10^{-16} \mathrm{~m}$.

And let the momentum $P$ defined as;

$P r_{c}=\hbar=m c r_{c}$. And let us incorporate this length in our equality:

$$
\left(k \frac{e^{2}}{r_{o}}\right)\left[\frac{r_{o}}{r_{c}} \cdot \frac{p}{\left(\frac{n}{p} \cdot p\right)^{1 / 3}}\right]=\left(-G C_{o} \frac{m_{o}^{2}}{r_{o}}\right)\left(\frac{r_{o}}{r_{c}}\right) n^{2 / 3}
$$

This density allows us to use a new expression as Compton sphere instead of Fermi one. In GCU and because of $n \gg p$ Pauli term of Compton sphere appears as predominant kinetic energy. For each neutron we have the average $u_{c}=$

$$
=u_{c}\left(A-2 Z^{2}\right) / A=u_{c}(n-2 p) / n=u_{c}
$$

In Compton sphere; Pauli Exclusion Principle needs to multiply the number of the filled states by two (+/- spin) and to divide over eight [5] to get only the positive space octant (where $\sin -\mathrm{kx}=\sin \mathrm{kx}$ ) so we have;

The density $N_{s} / V=1 / V_{s}=n / V$

$$
u_{c}=\left(\hbar^{2} / 2 m\right)\left(3 \pi^{2} N / V\right)^{2 / 3} .
$$

For all neutrons; 


$$
=\int u_{c} d N=\frac{\hbar^{2}}{2 m}\left(\frac{3 \pi^{2}}{V}\right)^{2 / 3} \int(N)^{2 / 3} d N=(3 / 5) u_{c} N \text {. And }
$$

finally the average $\left\langle u_{c}\right\rangle=(3 / 5) u_{c}$. By putting $r_{c} \approx 2 \times$ $10^{-16} \mathrm{~m}$ and substitution we can estimate the average $<u_{c}>$

$$
\approx 1.5 \times 10^{-10} j=m_{o} c^{2}
$$

The relativistic gravitational potential $u_{g}(V)$ (as speed function) would be affected by $v$ more than the quantum term field $u_{c}(V)$ such that $1 / c_{o}=\left[1-\left(V^{2} / c^{2}\right)\right]$ would appear in the denominator of $u_{g}(V)$ with power 3/2 while it appears in the denominator of $u_{c}(V)$ only with power $1 / 2$

$u_{c}$ would appear as;

$$
u_{c}=A n^{2 / 3} \frac{c_{o}}{r_{o}^{2} m_{o} c_{o}^{1 / 2}}
$$

And, $u_{G}=-B n^{2 / 3} m_{o}^{2} c_{o} \frac{c_{o}{ }^{1 / 2}}{r_{o}}$

Where, $A$ and $B$ are constants and $r_{o}=r_{c}$.

On equality -at the speed $V$ approaches $c$ - and putting $m_{o}^{2}=-m^{2}$ we would have the equality;

$$
\left(A n^{2 / 3} / r_{o}^{2} m_{o}\right)=B n^{2 / 3} m_{o}^{2} c_{o} / r_{o} .
$$

And from equation (16) we get;

$$
\left(A n^{2 / 3} / r_{o}^{2} m_{o}\right)=B n^{2 / 3} m_{o}^{2} c_{o} / r_{o}=m c^{2} .
$$

And by adding the two inertia;

$$
u=m c^{2}+m c^{2}=m 2 c^{2}
$$

Which realizes the state $V^{2}=2 c^{2}$ as a relativistic solution for;

$m_{o}^{2}=-m^{2}$ where the negative sign gives the same radical physical meaning such that it is the operator which with this defined $V^{2}$ or definitely with this defined density. The equality of equation 18 could be written as;

$A n=B\left(n c_{o}\right) m^{3} r$ where it shows the side of view of a person such that he sees only the macroscopic event and so he understands the radius of the sphere $r$ from the side of view of the macroscopic reproductive function (under references frame transformation) as; $n c_{o}=N$.

Now we can notice that the constant in-between distance; $\left(r_{c}=2 \times 10^{-16} \mathrm{~m}\right)$ compelled each of the kinetics $u_{c}$ and $u_{G}$ to behave as inertial rest energy. Again like what we did in the potential (inertial) well of the proton we do also here. So the modification is to avoid the refused solution of $V=c$. So put $r_{c}=2 \times 10^{-16} m+\Delta r$ to obtain $V^{2}=2\left[c^{2}-\right.$ $\left.\left(c^{2} \frac{n}{p \times 1.29 \times 10^{36}}\right)\right] \approx 2 c^{2}$. So in all above we have (instead of the equality) to put the approximate equality $(\approx)$.

You can notice that the global strategy of our manipulation is that we began with a speed $\mathrm{v}=\mathrm{c}$ the we modified it to $\mathrm{v} \approx \mathrm{c}$ then we put more modification as a defined $\mathrm{v}$ as; $V^{2}=c^{2}-\left(c^{2} \frac{n}{p \times 1.29 \times 10^{36}}\right)$ and then instead of saying that $V$ is close to $c$ we say that; the in-between distance is close to $r_{c}$ as a state of existence or actually as Gods state. On other words up till now we do not study how to get such high speed or such close distance but we say that if such a state is exist then what is its physics. In separate chapters we will find that actually such state had existence in a stage of stages of evolution of the solar system where it left behind many of parameters in the solar system as documents for this proposal. The final modification is to say that instead of the above- all these microscopic events are initiating by the idea that; we may have a particle with cut energy in the form of;

$$
\left(m_{o} c^{2}-\Delta m_{o} c^{2}\right)
$$

The neutron (like the proton) appears in its potential (or precisely, the inertial) well as a pair of conjugate. Here we have to notice that the state of the speed approaches $\mathrm{c}$ is a description of production of one single $u$ of the pair while

$V^{2}=2 c^{2}$ is a description of the two $\mathrm{u}$ of the pair. We have also to notice that the protons have the same wavy behavior and so it has -also- $u_{c}$.

But this term could be neglected because of the distance between two neighbor protons $=10^{-4} r_{c}$ (where $n / p$ as we will see is about ten powers twelve). Any way this term can appear (although it is comparatively small) during estimating $\mathrm{p}$, of GCU as;

$$
m_{o} c^{2}=k e^{2} q / r \text {. where; } q\left(1-10^{-4}\right)=p=q .
$$

The average $u$ inside Compton sphere and the factor 5/3; We estimated the average $u_{c}=1.5 \times 10^{-10} j=m c^{2}$. From equation 3 , the average $u_{G}$ and the average $u_{e}$ is;

$$
<u_{G}>=<u_{e}>=\frac{3}{5} m_{o} c^{2}
$$

This form is not compatible with the inertial form $m_{o} c^{2}$. So we have to think in a manipulation returns the equations after integration to the general inertial form.

$$
\left[\left(\frac{k e^{2}}{r_{o}}\right) \frac{r_{o}}{r_{c}}\right] \frac{p^{2 / 3}}{\left(\frac{n}{p}\right)^{1 / 3}} d p=\left[G C_{o} \frac{m^{2}}{r_{o}} \cdot \frac{r_{o}}{r_{c}}\right] n^{2 / 3} d p
$$

Put the big brackets of the left and right sides to equal A and $\mathrm{B}$ respectively;

$$
\begin{gathered}
\frac{A}{\left(\frac{n}{p}\right)^{1 / 3}} \int p^{2 / 3} d p=B \int\left(\frac{n}{p} \cdot p\right)^{2 / 3} d p \\
=B\left(\frac{n}{p}\right)^{2 / 3} \int p^{2 / 3} d p \\
A \int p^{2 / 3} d p=B\left(\frac{n}{p}\right) \int p^{2 / 3} d p \\
\frac{A}{\left(\frac{n}{p}\right)^{1 / 3}} \int p^{2 / 3} d p=B \int\left(\frac{n}{p} \cdot p\right)^{2 / 3} d p \\
=B\left(\frac{n}{p}\right)^{2 / 3} \int p^{2 / 3} d p \\
A \int p^{2 / 3} d p=B\left(\frac{n}{p}\right) \int p^{2 / 3} d p
\end{gathered}
$$




$$
\begin{gathered}
\frac{3}{5} A \cdot p^{2 / 3} \cdot p=\frac{3}{5} B \frac{n}{p} p^{2 / 3} \cdot p \\
A \cdot p^{2 / 3} \cdot p=\frac{3}{5} B\left(\frac{5}{3} \frac{n}{p}\right) p^{2 / 3} \cdot p \\
k \frac{e^{2}}{r_{o}} \frac{r_{o}}{r_{c}}=\frac{3}{5} G c_{O} \frac{m^{2}}{r_{o}} \frac{r_{o}}{r_{c}} \frac{n}{p}
\end{gathered}
$$

Put the square bracket of the right side as; $\left(\frac{5}{3} \frac{n}{p}\right)=\bar{n} / p$.

Divide both sides over $\mathrm{p}$ and factorize the factor $\bar{n} / p$ into two factors one with power third and put it in the denominator of the left side and the other with power two thirds and leave it at site as;

$$
\begin{gathered}
A(p)^{2 / 3} /\left(\frac{\bar{n}}{p}\right)^{1 / 3}=\frac{3}{5} B[(\bar{n} / p) p]^{2 / 3}=\frac{3}{5} B(\bar{n})^{2 / 3} \\
<u_{e}>=A(p)^{2 / 3} /\left(\frac{\bar{n}}{p}\right)^{1 / 3}=m_{o} c^{2} \\
<u_{G}>=\frac{3}{5} B(\bar{n})^{2 / 3}=m_{o} c^{2} . \text { Where } \bar{n}=\frac{5}{3} n \text { and } \\
\bar{n} / p=\left(\frac{5}{3} \frac{n}{p}\right) .
\end{gathered}
$$

Once GCU is constructed and it is coming to - physicallygrow there would be retardation of the role of $u_{c}$ which is constant and there would be progress of the role of each of $u_{e}$ and $u_{G}$. The additive inertias should be controlled by the equality relation of equation from which we can see that each of $\mathrm{A}$ and $\mathrm{B}$ are constants so; $\frac{n}{p}$ should be forever constant so let us remember equation 20 as;

$A p^{2 / 3} p=B \frac{n}{p} p^{2 / 3}$. Kindly note that we mean with the factor $\frac{n}{p}$ is that constant which we will define its value in a separate chapter, but other than this and all over the study and in all the estimations the ratio between $n$ and $p$ would equal $\left(\frac{5}{3} \frac{n}{p}\right)$.

Now the mother sphere $S_{1}$ was formed of $p$ and $n$ with the constant $\frac{n}{p}$. In the next stage (sphere $S_{2}$ ) each particle appeared as; the original one and the added two as;

$S_{2}=3 S_{1}$. Before we go on we can put the general idea of the physical growth;

- The energy is quantized as $m_{o} c^{2}$

- The proton potential well is replaced by proton inertial well in which the energy is expressed as addition of protons.

- $\quad$ For each added proton there would be in the neutron inertial well (from the above equality) addition of $\frac{n}{p}$ number of neutrons so the added particles are multiples of huge proton (one proton $+\frac{n}{p}$ neutrons).

Now in the next stage $\left(S_{3}\right)$ we would have; $u_{e}$ per each mother proton $=\left(k \frac{e^{2}}{r_{o}}\right)\left[\frac{r_{o}}{r_{c}} \cdot \frac{p^{2 / 3}}{\left(\frac{n}{p}\right)^{1 / 3}}\right](3)^{2 / 3}=(2.08) m_{o} c^{2}$ (you have to notice that the added fraction would disappear on collecting allover $\mathrm{p}$; so for simplification let us say that; $u_{e}$ for each proton $=(2) m_{o} c^{2}$.

This means; each proton of $S_{1}$ would become 3 and then in a next stage would become $3 \times 2$ protons. And since from the above equality each proton is preserved by $\frac{n}{p}$ neutrons so the added neutrons in this stage (for each added proton) are;

$(3)^{2 / 3} \frac{n}{p}=2 \frac{n}{p}$ neutrons (or you can see it as two neutrons are produced from each neutron). This means that;

Number of the particles of $S_{3}=6.08$ times that of $S_{1}$ or 2.08 times $S_{2}$.

And so on; each stage would use the output of the previous stage as its own input. The important notice here is to know how to compare between what occurs in the state of the unit giant charge (where we have electric energy per elementary charge equal $E_{e}$ which is equivalently conjugated with the relativistic gravitational energy per the same elementary charge $E_{G 1}$ and we have also due to the uncertainty -Pauli exclusion factor- energy per a neutron equal $E_{u}$ which is conjugated equivalently with the relativistic gravitational energy per the same neutron $E_{G 2}$ where $E_{G 1}=E_{e}=E_{u}=$ $E_{G 2}$ ) and what occurs in physics of the state of the growing giant charge where although the sphere adds particles yet the density $\mathrm{N} / \mathrm{v}$ is still conserved so we have the growing conjugate $E_{e}=E_{G 1}$ and we have the constant conjugate $E_{u}=E_{G 2}$. This is because the relativistic can realize the relative motions.

Now we can deduce that the giant charge is a little dark compact body -with a defined density- which although has great energy yet this energy is converted into fermions. This is why it does not degenerate further; because of at the range of Compton wave length the relativistic gravitational energy $\left(E_{G}\right)$ and its conjugates are transformed into fermions (replication).

The binding energy (the cut energy);

You cannot remove all the energy from a system. Heisenberg said[6].

Actually in our system $\Delta E$ appears as $\Delta m c^{2}$

We showed that our system respected the universal constant $\mathrm{c}$ and made the particles move with $V \approx c$. And then overcame the huge energy which had to be produced in such high speed such that the state $V^{2} \approx 2 c^{2}$ had guaranteed that the alternative solution for this energy is the production of multiples of the inertial $m c^{2}$. But this does not mean that we have zero binding energy.

The relation; $V^{2}=2\left[c^{2}-\left(c^{2} \frac{n}{p \times 1.29 \times 10^{36}}\right)\right]$ guaranteed that the subatomic particle which has $E_{o}=m_{o} c^{2}$ (as a singled particle outside our system) would have inside our 
system (as produced particle) inertial energy $=E_{o}$

$=m_{o}\left[c^{2}-\left(c^{2} \frac{n}{p \times 1.29 \times 10^{36}}\right)\right]$ and so we would have a cut of energy as; $\Delta E$

$=\Delta m c^{2}=m_{o}\left(c^{2} \frac{n}{p \times 1.29 \times 10^{36}}\right)=m_{o} c^{2} / c_{o}=u_{G} / c_{o}=u_{g}$

(such that $u_{g}$ is the ordinary gravitational potential without $c_{o}$ ). This term which appears in our system resembles the volume term $a_{v}$ of the liquid drop model (semi empirical mass formula) [7] of the ordinary nucleus. So we have potential binding energy as;

$$
U_{p}=\Delta m c^{2}+0+0 \ldots \ldots
$$

Actually this is the macroscopic equation which summarizes all the previous microscopic events where all the energies had appeared as particles production except a cut in the form of; $\Delta m c^{2}=m_{o}\left(c^{2} \frac{n}{p \times 1.29 \times 10^{36}}\right)=m_{o} c^{2} / c_{o}=$ $u_{g}$ which appears in this complete dark and rest sphere.

But we have to notice that this $\Delta m c^{2}$ is a cut from each of the produced particles and not from the mother one. Such a notice would give a respective consideration when we speak about a generation close to the mother sphere while would be inconsiderable with the far ones.

Defining the giant atom[8];

The giant charge $A$ with radius $\mathrm{r}$ to orbit another one $(-A=B)$ with a radius of orbit $=R$ needs the following condition;

Since the particles of $A$ (or $B$ ) are bind with each other by the average $E_{b}$ per a huge charge;

$$
E_{b}=\frac{3}{5}\left(\frac{m \cdot n}{r}\right)\left(\frac{n}{p} \cdot m\right)
$$

And since a huge charge e (such that each huge charge contains one elementary particle) of $A$ to orbit $B$ would have kinetic energy due to the mutual potential energy between $e$ (the quantity of the charge in a huge charge) and $p$ (number of the charges) of the sphere $B$;

$$
E_{k}=\frac{1}{2} k \frac{e p}{R} e
$$

When $A$ (as one body; not each charge alone) orbits $B$ (kindly look at appendix 2) it would be defined by;

$$
\begin{aligned}
& \frac{3}{5}\left(\frac{m \cdot n}{r}\right)\left(\frac{n}{p} \cdot m\right) d p \geq \frac{1}{2} k \frac{e p}{R} e \cdot d p \\
& \frac{3}{5}\left(\frac{m \cdot n}{r}\right)\left(\frac{n}{p} \cdot m\right) \int d p \geq \frac{1}{2} k \frac{e p}{R} e \int d p \\
& \frac{3}{5}\left(\frac{m \cdot n}{r}\right)\left(\frac{n}{p} \cdot m\right) \int d p \geq \frac{1}{2} k e \frac{e}{R} \int p d p
\end{aligned}
$$

If $n / p$ was constant then;

$\frac{3}{5}\left(\frac{m \cdot n}{r}\right)^{2} \geq \frac{1}{4} k(e . p) \frac{e \cdot p}{R}$. In our previous papers; the first named the giant atom like system and the second named the electrostatic energy in a rest form we took the solution of the equality such that the strategy of the giant atom is based on the least possible quantity of particles[8,9]

$$
\begin{gathered}
\therefore \frac{3}{5}\left(\frac{m \cdot n}{r}\right)^{2}=\frac{1}{4} k(e \cdot p) \frac{e \cdot p}{R}=\frac{1}{4} m V^{2} \\
V^{2}=2.4 G M N^{2 / 3} / r_{c}
\end{gathered}
$$

In the same papers we estimated the speed; $V=5 \times 10^{4} \mathrm{~m} / \mathrm{s}$. And the radius;

$$
R=6 \times 10^{10} \mathrm{~m} .
$$

Actually this is coincident with the parameters of the nearest planet (nearest for sun) [8,9]

In the same above mentioned papers we put a general system which proved existence of the giant atom as a historical stage in stages of evolution of the solar system and also we put a suggestion for how to create GCU.

Kindly go again to eq. 20 and rewrite it again in the next modified form;

$$
k e^{2} p / r=G m^{2}\left(n c_{o}\right) / r
$$

You notice outside the square bracket $m$ appears as $m_{o}$ while the square bracket realizes the alternative relativistic solution by multiplication the number $\mathrm{n}$.

Now let us define $\frac{n}{p}$ and $c_{o}$.

From the definition of $\mu$ and from equation 14;

$$
\mu=c_{o}\left(\frac{n}{p}\right)=\frac{N}{P}=1.29 \times 10^{36}
$$

We want to define $C_{o}$ as a universal constant. So to get it away from the parameters which are specific to GCU and so we would get it from general parameters rather than specific ones. So let us go on;

$$
V^{2}=2.4 G M N^{2 / 3} / r_{c}
$$

Where $V$ is the speed of the ground state of the orbit of any inversed atomic system[9]. As it is known that the proton rest mass is about 1840 time that of the electron so the latter needs 1840 times the number of the neutron to reserve it inside its giant electron as much as the first needs. So the giant proton is 1 over 1840 times the mass of the giant electron so this giant proton orbits the giant electron with the speed $V$ of the last equation as a complete circular orbit (but when we will estimate the radius of the orbit of the giant atom we would take in consideration the eccentricity of its specific elliptical orbit) ${ }^{(9)}$ Putting the magnitude of $\mathrm{v}$ in the above equation we can get;

$$
N=2.4 \times 10^{45} \text { neutron. }
$$

$\therefore$ The number of the protons $p$ inside the giant proton unit could be estimated from equations 14 and 28 as;

$$
\frac{N}{\mu}=p=1.85 \times 10^{9} \text { protons. }
$$

The constant $\mathrm{n} / \mathrm{p}$ could be estimated from the general law;

$$
\begin{gathered}
\left(k \frac{e^{2}}{r_{o}}\right)[1]=m_{o} c^{2} \\
\left(k \frac{e^{2}}{r_{o}}\right)\left[\frac{r_{o}}{r_{c}} \cdot \frac{p^{2 / 3}}{\left(\frac{n}{p}\right)^{1 / 3}}\right]=m_{o} c^{2} \text { where; }\left[\frac{r_{o}}{r_{c}} \cdot \frac{p^{2 / 3}}{\left(\frac{n}{p}\right)^{1 / 3}}\right]=[1]
\end{gathered}
$$




$$
\frac{n}{p}=1.15 \times 10^{12}
$$

And finally from eq. 14 and 30 we can estimate the constant;

$$
c_{o}=1.29 \times 10^{36} / 1.15 \times 10^{12}=1.08 \times 10^{24} .
$$

The idea of the state $V^{2}=2 c^{2}$ as a synonymous for particles production such that the daughter one has the same inertial mass of the mother one or $m=m_{o}$ makes us go far to give a physical relativistic simulation for self-replication. $V=c$

\section{Replication of the giant neutron unit}

Here let us now remember that a macroscopic state in the form of $V^{2}=2 c^{2}$ and equality in the form of equation 16 are required with a microscopic event defining the speed $V \approx$ $c$. Each of these three conditions has its role as follows;

The state $\left(V^{2}=2 c^{2}\right)$ gives the meaning of production by copy and paste or in the relativistic expression it means $m^{2}=-m_{o}^{2}$.

Where the negative sign is vanished in the equality and consequently opens the way for the symmetric addition to realize the state; $m v^{2}=m 2 c^{2}$ and also to realize the productive function as; $m V^{2}=2 m c^{2}$. While the third condition would determine how many copies we would get or generally it defines the shape of this function.

We can summarize and say that; the state $\mathrm{v} \approx \mathrm{c}$ as an odd state is the microstate which appears as motive inertial reference frame giving on the scope of special relativity slaw dilated time (the rest time multiplied by the equality facto co) where on this relativistic slaw time, the number of the particles $\mathrm{N}$ appears as; $\mathrm{N}=\mathrm{n}$ co. The other face of the coin is the rest reference frame $(\mathrm{v} 2=2 \mathrm{c} 2)$ which appears as the even or the conjugate rest state where this rest time translates the state; $\mathrm{v} 2=2 \mathrm{c} 2$ into two particles.

Let the neutrons of the giant neutron unit get in a contact state (Gods state) defined by $r=r_{c}$ which gives from equations 15 (where you notice that $r^{2}$ appears in the denominator of $u_{c}$ ) and 16 (where $c^{2}$ appears);

$u_{c}=1.5 \times 10^{-10}=m c^{2}$. And again we recall all the scientific relativistic basis of the microscopic events to get the equality with $u_{G}$ and then the symmetric addition as;

$u_{c}+u_{G}=m c^{2}+m c^{2}=m 2 c^{2}$ which realizes the state $V^{2}=2 c^{2}$. Or in the form of;

$u_{c}+u_{G}=m c^{2}+m c^{2}=2\left(m c^{2}\right)$ which means two neutrons are added to the mother one.

Suppose we have two neutrons near to each other such that each of the relativistic and the quantum wavy effect is considerable. Put;

$$
\beta^{2}=\frac{V^{2}}{c^{2}} \text { and } 1 /\left(1-\beta^{2}\right)=\mu
$$

The quantum behavior appears as;

$$
p^{\lambda}=\mathrm{h}
$$

Putting $r_{c}=2 \times 10^{-16}$ meter and $m=1.66 \times$ $10^{-27}$ kilogram;
From above and from (33); $V=c$

From equation 16 put $E_{c}=m_{o} c^{2}$ and the equality needs;

$$
\begin{aligned}
& m_{o} c^{2}=G m_{o}^{2} \mu / r_{c} \\
& \therefore \mu=1.6 \times 10^{38}
\end{aligned}
$$

For a population of neutrons $\mathrm{n}$ with the same density we would have

$$
E_{c} n=n m_{o} c^{2}=\frac{3}{5} m^{2}\left(n c_{o}\right) n^{2 / 3} / r_{c}
$$

Here you see the term n. $c_{o}$ appears as a conjugate and so it looks like the modified form of equation (20). And also you notice that $\mathrm{m}$ appeared versus $m_{o}$ while the alternative relativistic solution appears as multiplication of $n$ by the speed function factor $c_{o}(v)$.

$$
\begin{aligned}
\therefore & \frac{3}{5} c_{o} n^{2 / 3}=\mu=1.6 \times 10^{38} \\
& c_{o}=2.8 \times 10^{38} / n^{2 / 3}
\end{aligned}
$$

You can see $c_{o}$ of the reproductive function differs in the form from that of GCU and it depends on the arbitrary variable $n^{2 / 3}$

Now to realize the equality of equation 35 put the speed of $u_{c}=V_{c}$ in a form like eq. 2 as;

$\left(V_{c}\right)^{2} \approx c^{2}=c^{2}-c^{2} / c_{o}$

The global solution is formed of;

$1-m=m_{o}$

2- $\quad N=2 n$ (at infinitesimal $\Delta t$ ). This occurs by putting $V^{2} \approx c^{2}$ where the output appears as duplication of the giant neutron unit.

3- $N_{g}=c_{o} n$ (at $\sum \Delta t$ ). The output appears as the continuous growth of the unit throughout the time. Of course the cut term which appears after the minus in eq. 37 allows a binding energy in the form of $m c^{2} / c_{o}$ per a produced particle which can take the form of; $E_{g} \cdot c_{o} / c_{o}=E_{g}$.

Can we create a reproductive function of the giant neutron unit?

Of course we cannot bring particles with in-between distance $=u_{c}$ and it is not easy to bring them in the density of the reproductive function. So it may be formed by recalling the corresponding circumstances. So let us bring neutrons such that their average speed equals the average speed of the particles of the reproductive function as $V^{2}=c^{2} / c_{o}$. And if we have number of neutrons equal about $10^{21}$ which gives $n^{2 / 3}=10^{14}$

And then: $c_{o}$ of the reproductive function $=2.8 \times$ $10^{38} / 10^{14}=2.8 \times 10^{24}$.

$$
\therefore V=\sqrt{\left(c^{2} / c_{o}\right)} \approx 3.5 \times 10^{-8} \mathrm{~m} / \mathrm{s} .
$$

From de Broglie equation this speed gives; $r \approx 0.0002 \mathrm{~m}$.

So if we could to collect this quantity of neutrons in a defined evacuated volume such that $R=0.0002 \times n^{1 / 3}$ then I suggest that the collection would appear as reproductive function and the particles would get (by 
correspondence principle and not by gradual approach) the simultaneous state of $r=2 \times 10^{-16}$ meter. It is easy to conclude that $R$ is n-independent such that; $R=2000$ meter.

\section{Conclusions}

Physics of the giant atom like system is a scientific truth. This is confirmed by that we found complete coincidence between the parameters of such a system with that of the solar system.

The scientific value of this mathematic study is that it discovers the relativistic basic relation between the two long range acting forces and the inertial mass. Explicitly we can say that; each of the relativistic gravitational and the electrostatic energy can appear as inertial energy in the form of $m_{o} c^{2}$ minus a little cut in the form of $m \frac{c^{2}}{c_{o}}$ which realizes the reproductive function and describes physics of the giant atom.

Our study put also a suggestion for how to create a reproductive function.

\section{Appendix 1}

One may doubt and say that $\Delta \mathrm{E}$ is not equal $\left(k \frac{e^{2}}{r_{o}}\right)$ but it has the form of;

$\Delta \mathrm{E}=\frac{1}{2}\left(k \frac{e^{2}}{r_{o}}\right)[1]=\frac{1}{2} m_{o} c^{2}$. Never mind. let us go on to estimate $\Delta \mathrm{E}$. Actually Lorentz postulated that the energy of the charged particle is due to its electromagnetic behavior. So let us estimate the part of the energy due to the electric field and add it to that part due to the motion (spin) of the charged particle (magnetic energy). Now in each infinitesimal volume we have the electric energy density;

$(\Delta \mathrm{E})_{1}=\omega(E . E) / 2$. Where $\omega$ is the permittivity constant of space and $(E . E)$ is the dot product of the electric field vector (the result is scalar). And all over the spherical volume we have the electric potential per particle;

$u_{e}=\frac{e^{2}}{32 \pi^{2} a^{4} \omega} \int_{0}^{2 \pi} \int_{0}^{\pi} \int_{r}^{\infty} r^{2} \sin \theta d r d \theta d \phi=\frac{e^{2}}{4 \pi \omega(2 a)}=$ $\frac{1}{2} m_{o} c^{2}$.

The magnetic part of the energy due to spin;

We would estimate the magnetic field $B$ in $\mathrm{z}$ - direction (in S.I. units) postulating a literal circular motion of the charged particle with radius of rotation equals its classic radius $a=r$. This postulated motion is coincident with the method by which we estimated the electric part of the energy otherwise you cannot doubt in equation1). So we would estimate the magnitude of $B$ in $\mathrm{z}$ - direction due to motion of the charged elementary particle in a circle with length $l=2 \pi$ a where $a=r$. From Biot-Savart law; $B=$ $\frac{\mu}{4 \pi} i \frac{\int d l}{a^{2}}=\frac{\mu i}{2 r}$ where $\mu$ is the permeability constant of the space and $i$ is the electric current of the charged particle as; $i=\frac{e}{T}=\frac{e v}{2 \pi \mathrm{a}}$

$\therefore B=\frac{\mu e v}{4 \pi a^{2}}$. The magnetic moment $\mathrm{m}$ is estimated as;

$m=i \pi a^{2}$

$\therefore$ The energy part due to spin;

$u_{s}=m \times B$ where $\times$ is the cross product which gives in z- direction;

$$
=\frac{v^{2}}{4 \pi 2 a} e^{2} \mu .
$$

Put $v=c$ and put $\omega \mu c^{2}=1$

$\therefore u_{s}=\frac{e^{2}}{4 \pi \omega(2 a)}$. Or we can estimate it directly from Maxwell [10] equations as;

$$
(\Delta \mathrm{E})_{2}=B^{2} / 2 \mu \text {. Put } B=-E / c \text {, and } \omega \mu c^{2}=1
$$

$\therefore(\Delta \mathrm{E})_{2}=E^{2} \varepsilon / 2$. And allover the volume gives the same above result.

And the total energy is estimated by adding the two types of energy as;

$$
\therefore \mathrm{u}=\Delta \mathrm{E}=2 \frac{e^{2}}{4 \pi \omega(2 a)}=m_{o} c^{2}
$$

\section{Appendix 2}

It is easy to understand that if the total gravitational binding energy between the elementary particles inside the sphere was just equal or more than the attractive total electric energy between the two spheres then the sphere $A$ would orbit the sphere $B$ as;

$$
\frac{3}{5}\left(\frac{m \cdot n}{r}\right)\left(\frac{n}{p} \cdot m\right) \cdot p \geq \frac{1}{2} k \frac{e p}{R} e \cdot p
$$

Actually this equation is not accurate and so we need the next discussion;

Let the amount of the elementary particles of the sphere $A$ (the electrons) to be labeled $p_{i}$ where $i=1,2,3 \ldots \ldots \ldots . p$

And let the summation of the elementary particles of the sphere $B$ (the protons) to equal $P$, where $P=p$.

If the last equation was not obeyed then the charges of the two spheres would be attracted to each other as elementary particles to form atoms.

On the first event when the first electron $\mathrm{p}_{1}$ attracted into the sphere $B$, the attractive electric energy $E$, would be proportional with $P$.

On the second event when the second electron $\mathrm{p}_{2}$ attracted to $B$, the electric energy would be proportional with $(P-1)$ because of the previous event have transformed one proton of $B$ into atom. And so on each event would subtract a proton till we reach to the last event where the sphere $B$ contains one proton only. Finally we would have the summation of the series as;

$$
\sum E=k(e \cdot p / R) p / 2=k(e / R) p \int d p
$$

Now we reached to the point that the two spheres to be 
attracted toward each other (as spheres not as elementary particles) we need;

$$
\frac{3}{5}\left(\frac{m \cdot n}{r}\right)\left(\frac{n}{p} \cdot m\right) \cdot p \geq k(e / R) p \int d p
$$

So the two spheres to orbit each other we need the form of equations 25 and 26 .

The last notice here is that; actually the elementary particles of the sphere A are not pulled to the other sphere as singles but actually as shots; the superficial ones then the deeper but finally we can define the series;

$$
\begin{gathered}
(P-a)+(p-b)+(p-c) \ldots \ldots \ldots+c+b+a=\frac{p^{2}}{2} \\
=p \int d p .
\end{gathered}
$$

\section{REFERENCES}

[1] Albert Einestein (2001), Relativity; the special and general theory. Reprint of 1920 translation by Robert W. Lawson Ed.

[2] David J. Griffiths, Introduction to quantum mechanics, Prentice-hall, 1995, p 155.

[3] Alzergin and v. Korepin, Letter in mathematical physics, vol. 6, 1982

[4] Garay Luis J, Quantum gravity of minimum length. International journal of modern physics (1995).

[5] Alan Doolittle, Density of states and Fermi energy concept.

[6] Wemer Heisenberg, The physical principle of quantum theory

[7] Bailey,D. Semi empirical nuclear mass formula: Strings and binding energy 2011.

[8] Eldieb Emad, The electrostatic energy in a rest form; evidences and recommendations, international research journal of pure and applied physics, vol. 3, No. 1 June. 2015.

[9] Eldieb Emad, The giant atom like system, advanced studies in theoretical physics vol. 3, 2009.

[10] Claus W. Turtur, An explanation of electron mass by the energy of its fields, university of applied sciences Braunchweig-Wolfenbuttel, Germany. 